\title{
Skill and reliability of climate model ensembles at the Last Glacial Maximum and mid-Holocene
}

\author{
J. C. Hargreaves ${ }^{1}$, J. D. Annan ${ }^{1}$, R. Ohgaito ${ }^{1}$, A. Paul $^{2}$, and A. Abe-Ouchi ${ }^{1,3}$ \\ ${ }^{1}$ RIGC/JAMSTEC, Yokohama Institute for Earth Sciences, Yokohama, Japan \\ ${ }^{2}$ University of Bremen, Bremen, Germany \\ ${ }^{3}$ AORI, University of Tokyo, Tokyo, Japan \\ Correspondence to: J. C. Hargreaves (jules@ jamstec.go.jp)
}

Received: 20 July 2012 - Published in Clim. Past Discuss.: 13 August 2012

Revised: 22 February 2013 - Accepted: 22 February 2013 - Published: 21 March 2013

\begin{abstract}
Paleoclimate simulations provide us with an opportunity to critically confront and evaluate the performance of climate models in simulating the response of the climate system to changes in radiative forcing and other boundary conditions. Hargreaves et al. (2011) analysed the reliability of the Paleoclimate Modelling Intercomparison Project, PMIP2 model ensemble with respect to the MARGO sea surface temperature data synthesis (MARGO Project Members, 2009) for the Last Glacial Maximum (LGM, 21 ka BP). Here we extend that work to include a new comprehensive collection of land surface data (Bartlein et al., 2011), and introduce a novel analysis of the predictive skill of the models. We include output from the PMIP3 experiments, from the two models for which suitable data are currently available. We also perform the same analyses for the PMIP2 mid-Holocene (6 ka BP) ensembles and available proxy data sets.

Our results are predominantly positive for the LGM, suggesting that as well as the global mean change, the models can reproduce the observed pattern of change on the broadest scales, such as the overall land-sea contrast and polar amplification, although the more detailed sub-continental scale patterns of change remains elusive. In contrast, our results for the mid-Holocene are substantially negative, with the models failing to reproduce the observed changes with any degree of skill. One cause of this problem could be that the globallyand annually-averaged forcing anomaly is very weak at the mid-Holocene, and so the results are dominated by the more localised regional patterns in the parts of globe for which data are available. The root cause of the model-data mismatch at these scales is unclear. If the proxy calibration is itself reliable, then representativity error in the data-model comparison, and missing climate feedbacks in the models are other possible sources of error.
\end{abstract}

\section{Introduction}

Much of the current concern over climate change is based on decadal to centennial forecasts from climate models forced with increased greenhouse gas concentrations due to anthropogenic emissions. However, a direct assessment of the predictive performance of the models is not generally possible because the time scale of interest for climate change predictions is typically for decades or centuries into the future, so we cannot build up confidence and experience via repeated forecasts on a daily basis as is typical in the field of weather prediction. Therefore, in order to have confidence in the ability of the ensemble to provide a credible projection of future climates, we must try to develop other methods for assessing the performance of models in simulating climates which may be very different to today.

Paleoclimate simulations provide us with an opportunity to critically confront and evaluate the performance of climate models in simulating the response of the climate system to changes in radiative forcing and other boundary conditions. A particularly attractive feature of using paleoclimate simulations is that, in contrast to the situation regarding more recent climate changes, the performance of models over these intervals has not been directly used in their development. Therefore, these simulations provide a truly independent test of model performance and predictive skill under substantial changes in external forcing. The extent to which such assessments may then be used to imply skill for future forecasts is still, however, open to some debate, since not all the past climate changes are necessarily relevant for the future. Models which provide the most realistic simulations of past changes may not necessarily provide the most accurate predictions of future change. Nevertheless, 
the potential of such assessments to help in evaluating model performance provides strong motivation for research in this area. The sparse and semi-qualitative nature of paleoclimatic data (for example, interpretation as vegetation type) has motivated the development of advanced but semi-quantitative methods, such as using fuzzy logic to measure model-data mismatch (Guiot et al., 1999) and cluster analysis to classify types of model behaviour (Brewer et al., 2007). With the advent of new more comprehensive syntheses of gridded paleodata (MARGO Project Members, 2009; Bartlein et al., 2011), it becomes possible to attempt more directly quantitative analyses of model performance, which we undertake here.

The second phase of the Paleoclimate Modelling Intercomparison Project, PMIP2 (Braconnot et al., 2007a), established a common protocol of boundary conditions for two different paleoclimate intervals, the Last Glacial Maximum (LGM, $21 \mathrm{ka} \mathrm{BP}$ ) and the mid-Holocene (MH, $6 \mathrm{ka} \mathrm{BP})$. Of the two, the LGM represents by far the greatest change in climate with significantly decreased concentration of atmospheric carbon dioxide and other long-lived greenhouse gases, and large ice sheets over the northern hemisphere high latitudes. The climatic response was characterised predominantly by a large-scale cooling, albeit with substantial regional variation (Annan and Hargreaves, 2013). The forcing of the mid-Holocene is more subtle, with the only changes considered by the models being that of orbital forcing, and a moderate decrease in atmospheric methane. While this results in substantial changes in the seasonal and spatial pattern of the insolation, the annual mean forcing is rather small. Rather than globally homogeneous changes, the mid-Holocene experienced a number of more regional changes (Steig, 1999), with one of the the largest relating to shifts in monsoon patterns and the associated vegetation changes (Braconnot et al., 2007b).

In this paper we extend our previous work presented in Hargreaves et al. (2011), hereafter H11, which assessed sea surface temperature at the LGM. We use several state of the art proxy data syntheses for surface temperatures for both the LGM and $\mathrm{MH}$, and compare them to outputs from the coupled atmosphere and ocean (AOGCM) and coupled atmosphere, ocean and vegetation (AOVGCM) general circulation models in the PMIP2 database. For the LGM we additionally include the two models from the ongoing PMIP3 experiment for which sufficient output are available at the time of writing. We perform analyses based on quantitative model evaluation methods which are widely used in numerical weather prediction. We first present a rank histogram analysis to indicate reliability. Secondly, we introduce a skill analysis using two different reference baselines. We also present Taylor diagrams (Taylor, 2001). These diagrams summarise three conventional statistics, and have been widely used to analyse climate model ensemble output in the context of the modern climate. In addition, we introduce some simple modifications to these conventional statistics to account for observational uncertainty.

We introduce the models and data used in the analysis in Sect. 2. Then we overview the methods for analysis of reliability and skill in Sect. 3. In Sect. 4 we present the results from the LGM and MH, and this section is followed by the discussion and conclusions.

\section{Background to the model runs and proxy reconstructions}

\subsection{Last Glacial Maximum}

All the PMIP2 models analysed in this paper are either physical coupled climate models comprising atmosphere, ocean and sea ice components (AOGCMs), or additionally including vegetation modules (AOVGCMs). One of the models, ECBILT, is an intermediate complexity model (see Table 1). For the LGM, the forcing protocol (Braconnot et al., 2007a) comprises a set of boundary conditions including large northern hemisphere ice sheets, altered greenhouse gases including a reduction to $185 \mathrm{ppm}$ for atmospheric carbon dioxide, a small change in orbital forcing, and altered topography. There are some minor changes in the multi-model ensemble compared to our previous analysis of this ensemble in H11. Firstly, the output of the run from IPSL has been updated. In addition, previously the number of days in each month (which differs across models) was not taken into account when calculating the annual mean from the monthly data. This has been corrected, making a small difference to the values of the annual mean obtained. We require both surface air temperature (SAT) and sea surface temperature (SST) for our main analysis, and therefore only use the 9 models in the database for which both these variables are available. The boundary conditions for the PMIP3 experiments (see http://pmip3.lsce.ipsl.fr/) are slightly revised, primarily in respect of the ice sheet reconstruction, but remain sufficiently similar that it seems reasonable to also include these models where possible. We therefore also include two models from the PMIP3 experiments for which SAT and SST outputs were available. Thus we have a total of 11 models.

Where centres have contributed more than one model to our ensemble, there are reasons to expect that these models will be particularly similar to each other (Masson and Knutti, 2011) but there is little consensus about how to treat this. We start from the premise of assigning equal weight to each model. However, including two model versions that are identical to each other would be clearly pointless and indeed harmful as it would be equivalent to double-counting one model and would therefore reduce the effective sample size of our ensemble, degrading the results. The only difference between MIROC3.2 and MIROC3.2.2 (which are both in the PMIP2 database) is that one minor bug has been fixed 
in the latter, so we included only the latter model in the ensemble. For substantially updated versions of the same model (such as might exist in consecutive iterations of the PMIP experiments) or $\mathrm{AO}$ and $\mathrm{AOV}$ versions we would expect the differences to be rather greater. But, ideally we should wish for each new model included in the ensemble, to be not particularly more similar to one existing model than it is to all the others. Therefore, although our main analysis assigns equal weight to each model, we also test whether our results are changed by downweighting or excluding some particular sets of models in an attempt to compensate for model similarity.

The proxy data compilations that we use are largely based on two recent syntheses of land (SAT) and ocean (SST) data. The ocean data is primarily that of the MARGO synthesis (MARGO Project Members, 2009), but with a small number of points having been updated by Schmittner et al. (2011). These updated points may not be fully homogeneous with the original MARGO data set as the data error has not necessarily been estimated in an identical way. The land data is primarily the pollen-based compilation of Bartlein et al. (2011), again with some additions by Schmittner et al. (2011) which includes some data from ice cores. The Bartlein et al. (2011) data set may be considered a somewhat more ad hoc data set than the MARGO synthesis, in that the data and error estimates have been directly drawn from the original literature in which the underlying data were presented, rather than being recalculated homogeneously across the data set as in MARGO Project Members (2009). In addition, the temperature anomalies in Bartlein et al. (2011) are taken relative to the core tops in contrast to the modern World Ocean Atlas data that were used to anchor the MARGO anomalies. The SST data are analysed on the MARGO 5 degree grid, while all the SAT data are on a 2 degree grid. After removing grid points for which SST information is unavailable in one or more models (due to their differing land sea masks), there are 309 SST points left for comparison with PMIP2, and 300 points for comparison with PMIP2 + PMIP3. Our goal is to assess the model response to imposed forcing, rather than the forcing itself, so for the land data we remove those points for which $50 \%$ or more of the grid box area lies under the model's ice sheet. This affects 11 points, leaving 95 land points for both PMIP2 and PMIP3. Thus we have a total of 404 points for comparison with PMIP2 and 395 for comparison with the combined PMIP2 and PMIP3 ensemble.

Estimates of the data error uncertainty are included for all the data points, although we note that the MARGO errors are only defined in terms of their relative reliability. As in H11, we assume these values can be interpreted as Gaussian uncertainties in degrees Celsius, which gives an apparently plausible magnitude of uncertainties. The resulting errors range from $0.24{ }^{\circ} \mathrm{C}$ to $6.4{ }^{\circ} \mathrm{C}$ across the data set, with a large majority of values lying in the range $1^{\circ} \mathrm{C}$ to $2.5^{\circ} \mathrm{C}$. However, Annan and Hargreaves (2013) found some cause for concern in these error estimates and so we also test the sensitivity of our results to this. The model SST output was interpolated on to the 5 degree MARGO grid and the SAT onto the 2 degree Bartlein et al. (2011) grid. We use equal weighting for each grid box. The data and their uncertainty are shown in Fig. 1.

\subsection{Mid-Holocene}

For PMIP2 the mid-Holocene protocol includes only two changes compared to the pre-industrial climate. Orbital forcing parameters are changed, and the atmospheric methane concentration is decreased slightly (from $760 \mathrm{ppb}$ to $650 \mathrm{ppb}$ ). The changes in orbital parameters affect the seasonal pattern of insolation. Annually averaged, however, the insolation is everywhere very similar for pre-industrial and $6 \mathrm{ka}$, so we expect to see only a modest signal in the annual mean temperature field. Therefore, our primary interest is in changes in the seasonal cycle, although we also consider annually averaged temperature. There are 11 AOGCMs and 6 AOVGCMs in the PMIP MH ensemble that have both SAT and SST data available. Only one of the AOVGCMs, ECHAM, does not have a counterpart AOGCM in our ensemble (see Table 1). As well as the full ensemble, we also analyse a conservative ensemble where versions of the same model are downweighted (as discussed in Sect. 2.1) so that each underlying model has a total weight of 1 .

For the land temperatures at the MH we use the pollenbased data set of Bartlein et al. (2011). This synthesis includes estimates of annual average temperature as well as the temperatures of the hottest and coldest months, which indicate changes in the seasonal cycle. An uncertainty estimate is also included for all points, which ranges from $0.04{ }^{\circ} \mathrm{C}$ to $4.8^{\circ} \mathrm{C}$ over all the variables. The values at the lower end of this range appear particularly optimistic, and so we also perform some sensitivity tests to investigate the robustness of our results to these values. The number of data points varies slightly between the different variables (between 615 and 638), and the data are very clustered with high density in Europe and North America. The data, and the data error for the hottest month are shown in Fig. 2.

The "GHOST" SST data set (Leduc et al., 2010) contains annual average estimates of annually averaged SST at both $6 \mathrm{ka}$ and core-top for only 81 sites, and, while recognising that the core top is not an ideal or wholly consistent reference point (as the dates, and therefore climates, represented by the core tops may vary across the data set), we nevertheless take the difference between these two values to represent the annually averaged $\mathrm{MH}$ temperature anomaly with respect to the pre-industrial climate. Seasonal SST data are, as yet, unavailable. Many of these points are quite close to the coast, and due to varying coastlines in the models, SST output from all models is available for only 42 points. Data uncertainties are not readily available so for this analysis we assumed a 1 standard deviation error of $2{ }^{\circ} \mathrm{C}$ for all the points, which is representative of the data uncertainty of the MARGO SSTs. 
(a)

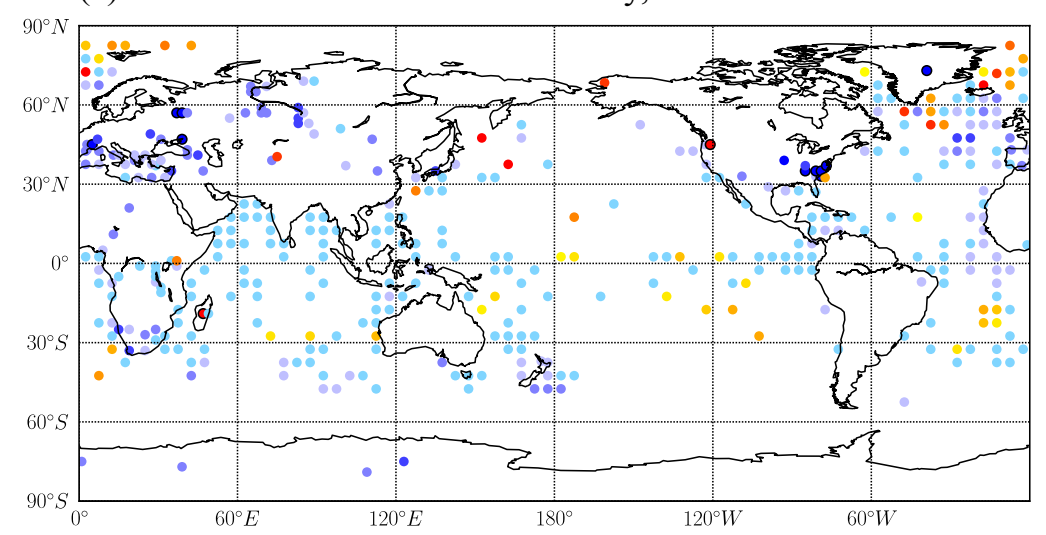

(b)

LGM anomaly, Data Uncertainty
LGM anomaly, Data

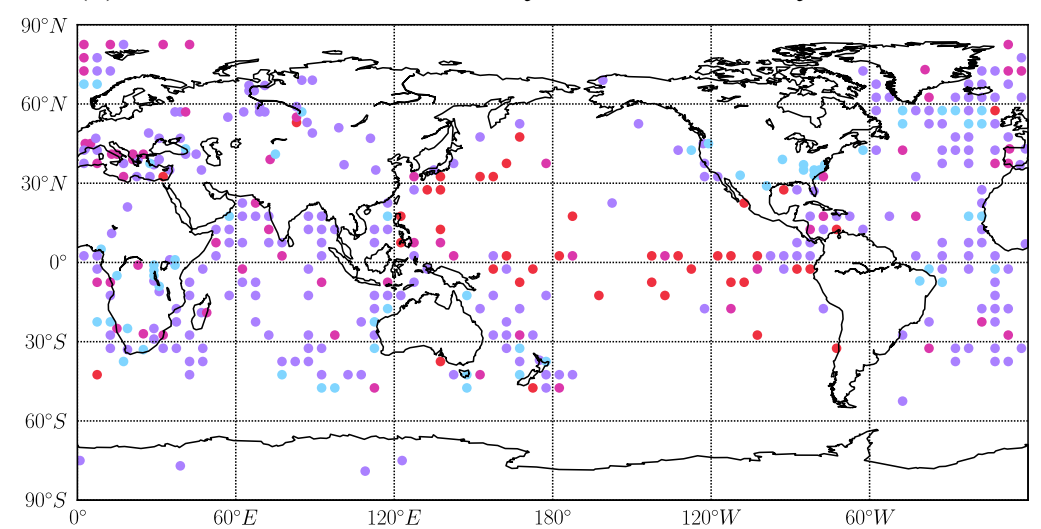

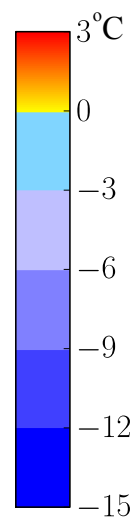

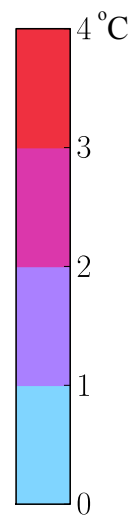

Fig. 1. (a) LGM temperature anomaly for the proxy reconstructions described in Sect. 2.1. The colorbar axes are chosen to best display the data. The actual minimum and maximum are $-16^{\circ} \mathrm{C}$ and $6.32^{\circ} \mathrm{C}$. (b) The value of the uncertainty on the annual mean included in the data synthesis. $\operatorname{Max}=6.42, \operatorname{Min}=0.24$ Mean $=1.73$.

Since we have no seasonal information for the ocean, most of our analyses are for the land only. These comprise the anomaly for the annual average, and the hottest and coldest months. Given the nature of the forcing, the change in the magnitude of the seasonal cycle would seem the most obvious target for the $\mathrm{MH}$, but there is some concern that calculating the anomaly of the hottest month minus coldest months may not provide a completely fair comparison with the data as the same proxies are not used to compile the two data sets. For the land and ocean together we analyse the annual average anomaly only.

It is clear that for the MH although we have more data points in total, the coverage is substantially less uniform than for the LGM and very sparse over large areas. As with the LGM analysis, we give equal weight to each data point. It is possible that the hottest month in the tropics may not be the same for the models and data due to the ways the calendars are configured (Joussaume and Braconnot, 1997). We have few data in the tropics, and the actual error in the value of the anomaly arising from this issue is expected to be small, so we do not expect this to have a significant effect on our results.

\section{Ensemble analysis methods}

\subsection{Reliability}

To assess the reliability of the ensembles we adopt the same approach used in several recent papers (Annan and Hargreaves, 2010; Hargreaves et al., 2011; Yokohata et al., 2011), in which we interpret the ensemble as representing a probabilistic prediction of the climate changes and assess its performance by means of the rank histogram (Annan and Hargreaves, 2010) formed by ranking each observation in the ensemble of predictions for each data point. In the case of a perfectly reliable ensemble (meaning that the truth can be considered as a draw from the distribution defined by the ensemble), the rank histogram would be flat to within sampling uncertainty. For an ensemble that is too wide such that the truth is close to the mean, the histogram is dome shaped. Conversely, an ensemble that is too narrow (often not including the truth) has a U-shaped rank histogram, with large values in one or both end bins. The analysis for the PMIP2 LGM ensemble using only the MARGO data set was already performed in $\mathrm{H} 11$, and overall produced encouraging results. In 
(a)

Data
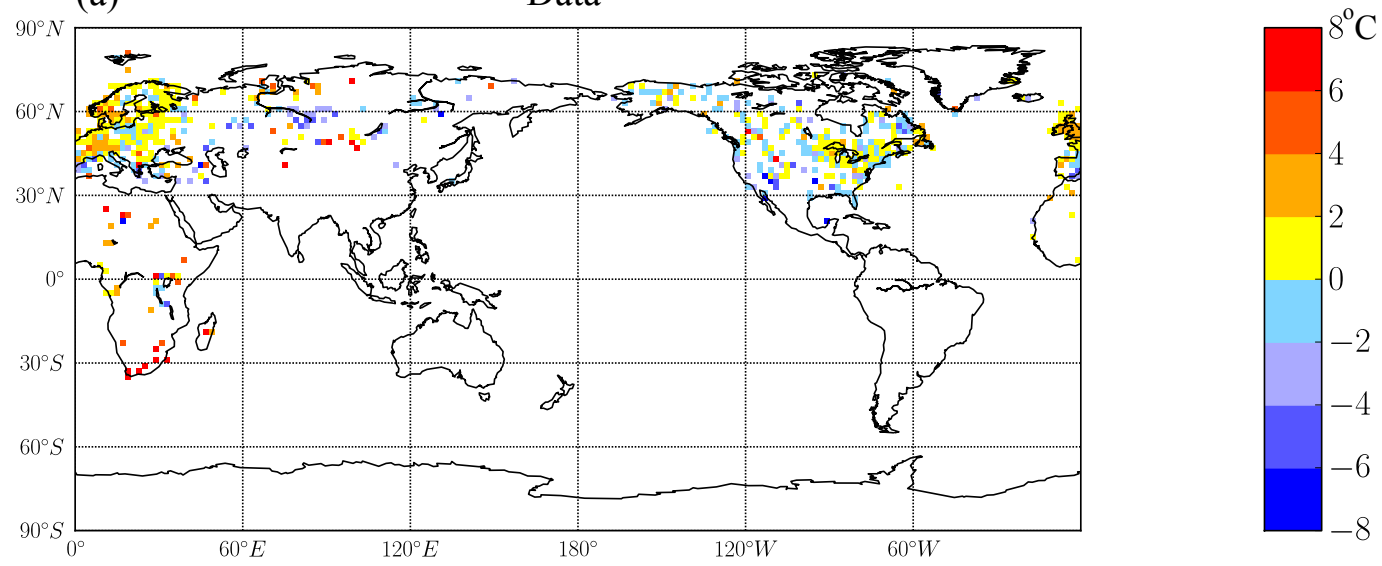

(b)

Data Error
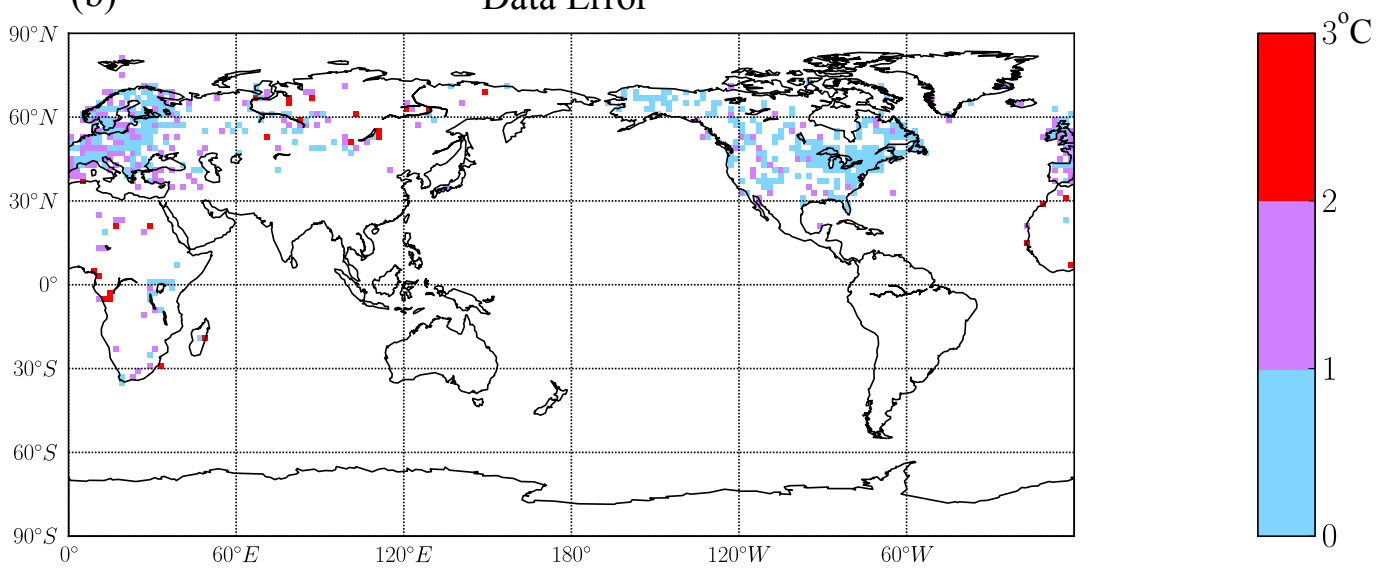

Fig. 2. Mid-Holocene temperature anomaly for the hottest month from the proxy reconstructions described in Sect. 2.2: (a) data, $\operatorname{Max}=10.0^{\circ} \mathrm{C}, \operatorname{Min}=-20.1^{\circ} \mathrm{C},(\mathbf{b})$ data error, $\operatorname{Max}=3.3, \operatorname{Min}=0.05$, Mean $=0.96$.

this analysis we extend this test by including land data. We also analyse the performance of the ensemble at the $\mathrm{MH}$, for which a larger ensemble of model simulations is available.

In order to make the models and data comparable, we follow the same procedure as H11 to account for data uncertainty, by adding random deviates (sampled from the assumed distributions of observational errors) to each model output before calculating the rank histogram. Thus, if reality was a sample from the model distribution, then the imprecise observation will also be a sample from the distribution of perturbed model outputs. We use the same statistical tests as H11 to quantify the significance of any divergence from flatness of the rank histogram (Jolliffe and Primo, 2008). The rank histogram test is only a necessary but not sufficient test of reliability, in that an ensemble which does not have a flat rank histogram may be considered unreliable, but a flat rank histogram does not necessitate reliability (Hamill, 2001). Indeed, with a large enough ensemble and fine data coverage we generally expect the ensemble to be unreliable at some level, and thus this test is not whether the ensemble is perfect, but rather whether the limitations are so substantial as to be immediately apparent with this standard test. In previous applications such as H11 and Yokohata et al. (2011), it has been found that the ranks of nearby observations are often highly correlated, and therefore the effective number of degrees of freedom are substantially lower than the number of data points. In order to estimate the number of degrees of freedom, we adopt the EOF analysis approach of Bretherton et al. (1999), as used by Annan and Hargreaves (2011). That is, rather than the conventional approach which typically uses a sequential series of fields, we calculate the EOFs of the ensemble of modelled equilibrium states. Analysing the LGM fields of SAT and SST for the model ensembles, we estimate a value of 8 degrees of freedom, which we use throughout our analyses. Our results are insensitive to reasonable changes in this value. 
Table 1. Overview of the model versions used in the different ensembles analysed. The names correspond to the filenames in the PMIP2 and CMIP5 databases.

\begin{tabular}{llllll}
\hline Model & PMIP2 LGM AOGCM & PMIP2 LGM AOVGCM & PMIP3 LGM & PMIP2 MH AOGCM & PMIP2 MH AOVGCM \\
\hline CCSM & CCSM3 & & & CCSM3 \\
CNRM & CNRM-CM33 & & & CSIRO-Mk3L-1.1 & \\
CSIRO & & & & ECBILTCLIOLOVECODE & ECBILTCLIOLOVECODE \\
ECBILT & ECBILTCLIO & ECHAM53-MPIOM127-LPJ & MPI-ESM-P & ECHAM53-MPIOM127-LPJ \\
ECHAM & & & & FGOALS-1.0g & FOAM \\
FGOALS & FGOALS-1.0g & & FOAM & UBRIS-HadCM3M2 \\
FOAM & & HadCM3M2 & GISSmodelE & \\
GISS & & & UBRIS-HadCM3M2a & \\
HadCM3 & HadCM3M2 & & & IPSL-CM5A-LR & \\
IPSL & IPSL-CM4-V1-MR & & MRI-CGCM2.3.4nfa & MRI-CGCM2.3.4nfa \\
MIROC & MIROC3.2.2 & & MRI-CGCM2.3.4fa & MRI-CGCM2.3.4fa \\
MRI-nfa & & & & \\
MRI-fa & & & &
\end{tabular}

*ECBILT is the only EMIC in the ensemble. All the other models are full general circulation models.

\subsection{Skill}

The concept of model skill in climate science has been occasionally touched upon, but it has rarely been clearly and quantitatively defined (Hargreaves, 2010). Here we use the term skill in the sense in which it is in common use in numerical weather prediction, which is as a relative measure of performance: skill compares the performance of the forecast under consideration, to that of a reference technique (Glickman, 2000). Perhaps the most straightforward of these is the skill score (SS) defined by

$\mathrm{SS}=1-\left(\frac{E_{\mathrm{f}}}{E_{\mathrm{ref}}}\right)$

where $E_{\mathrm{f}}$ is the error of the forecast under evaluation, and $E_{\text {ref }}$ is the error of the reference technique. A perfect forecast will have a skill score of 1 , one which has errors equal to that of the reference will have a skill score of 0 , and a negative skill score implies that the errors of the forecast are greater than that of the reference. It is conventional to describe a forecast with positive skill score as "having skill", but note that this is always defined relative to a specific reference. So for example, using a root mean square difference to evaluate the forecast, one has

$S=1-\sqrt{\frac{\sum_{i}\left(m_{i}-o_{i}\right)^{2}}{\sum_{i}\left(n_{i}-o_{i}\right)^{2}}}$,

where $m_{i}$ are the forecast results, $o_{i}$ the data and $n_{i}$ the reference. According to this equation, however, in the presence of error on the data, even a perfect model (where the model prediction precisely matches reality) would not achieve a skill of 1 . Especially in the case of paleoclimates, the uncertainty on the data is often substantial (Hargreaves et al., 2011), and must be taken into account for a fair evaluation. We therefore modify Eq. (1) as follows:

$$
S=1-\sqrt{\frac{\sum\left(m_{i}-o_{i}\right)^{2}-\sum\left(e_{i}^{2}\right)}{\sum\left(n_{i}-o_{i}\right)^{2}-\sum\left(e_{i}^{2}\right)}}
$$

where $e_{i}$ are the one standard deviation uncertainties on the observations. The numerator and denominator in the fraction have here been adjusted to account for the observational errors, under the assumption that these are independent of the forecast errors. Note that the skill score here becomes undefined if either the model or the reference agrees more closely with the data than the data errors indicate should be possible. Such an event would be evidence either that the data errors are overestimated, or else that the model had already been over-tuned to the observations. In principle, no model should agree with the data with smaller residuals than the observational errors, since even reality only just achieves this close a match, and only then if the observational errors have not been underestimated.

For the obvious reason that long-term forecasts are have not been generally realised in climate models, skill analyses of this nature for climate model predictions are rare. One simple analysis was performed by Hargreaves (2010), which indicated that, at least on the global scale, the $30 \mathrm{yr}$ forecast made by Hansen to the USA congress in 1988 had some skill. Unfortunately, regional data from this forecast were not available for testing. We are not aware of previous analyses of model skill for paleoclimates and it has not been established what might be an appropriate reference forecast for such calculations. In numerical weather prediction, persistence (that tomorrow's weather is the same as today's) is a common baseline for short-term forecast evaluation. However, for seasonal prediction, where persistence is clearly inappropriate, it would be more usual to use the seasonal climatology as a reference. An analogous reference for climate change predictions might be that the climate persists, that is, a reference of no change. It should be clear that this is a rather 
minimal baseline to beat, only requiring that the model predicts any forced response at each location to within a factor of anywhere between 0 and 2 times the correct amplitude (on average). On the other hand, it is worth checking, as it provides a baseline test as to whether the models are responding in a qualitatively appropriate manner. We might reasonably hope for our models to perform rather better than this, and provide a useful prediction not only of the overall magnitude, but also the spatial pattern of change. Thus we also employ a second reference to test the pattern of the change more directly. For this reference forecast, the climate change is assumed to be a uniform change equal to the mean change of the available data. This represents the case of a perfectly-tuned zero dimensional energy balance, in which the global mean temperature change is predicted which optimally matches the data, but without any information on the spatial pattern. In order to have skill with respect to this reference, the model must also represent the spatial pattern of change relative to this global mean.

\subsection{Conventional Taylor diagram analysis}

We also present an analysis of the model outputs in terms of the conventional statistics of (centred) RMS difference, correlation and field standard deviation which will be familiar to many readers. Such values are conveniently presented in a Taylor diagram (Taylor, 2001) which summarises these three values with a single point. The usual calculation and presentation of these statistics does not account for observational uncertainty, and Taylor (2001) only suggests investigating the effect that this might have on the results through the use of multiple data sets, which we do not have here. However, since we do have estimates of observational uncertainty, we can instead adjust the statistics to account for this. We present our results based on two approaches. First, we present the conventional results, without accounting for observational uncertainty, but also indicate where a hypothetical "perfect model" (which exactly matches the real climate system) would be located. This is straightforward to calculate (under the natural assumption that observational errors are independent of the climatic variables), as its normalised RMS difference from the imperfect observations will be just the root mean square of the observational uncertainties divided by the standard deviation of the observations, and the field standard deviation will add in quadrature to the errors to give the observational standard deviation, thus $\mathrm{sd}_{\text {real }}^{2}=\mathrm{sd}_{\mathrm{obs}}^{2}-\mathrm{sd}_{\text {errors }}^{2}$. We also present an alternative approach, in which we correct the statistics for each model, to indicate where they would be expected to lie relative to perfect observations. This is an equally simple calculation when error estimates are given, as for example the RMS modeltruth difference is calculated by subtracting (again, in quadrature) the estimated errors from the model-data differences.

\section{Results}

\subsection{Last Glacial Maximum analyses}

\subsubsection{LGM Reliability}

Figure 3 shows the reliability analysis for the combined LGM ensemble of 11 models for all the points at which we have data. Overall, the ensemble has a rank histogram which cannot be statistically distinguished from uniform (Fig. 3b), and the differences between the data and the ensemble mean (Fig. 3c) are mostly of similar magnitude to the uncertainty in the data. Looking at the map in Fig. 3a, there are some patches that are predominantly red or blue, indicating the spatial limit to the reliability. Analysing the ocean and land data separately (Fig. 4) we find that, assuming 8 degrees of freedom, the ensemble is statistically reliable with respect to both. However, we note that the histogram for the land (Fig. 4c) has a fairly large peak at the left hand side, indicating that the ensemble tends to have a greater anomaly than the data. It is also apparent that the difference between the ensemble mean and the data is larger for the land than for the ocean (Fig. 4d). This model-data difference exceeds the quoted data uncertainty much more frequently for the land than for the ocean.

Paleoclimate data are derived from measurements made from cores drilled into the surface of the earth at discrete locations. The open ocean may be considered laterally quite well mixed, whereas land has many more local features due primarily to high resolution topography. Thus it may be more difficult to derive a representative grid box average temperature from proxy data for direct comparison with the models over the land, than over the ocean. On the grid scale of the models, one sees more variation over land than ocean, but even so it is likely that the models have inadequate spatial variability at this scale due to smoothing in the forcing, boundary conditions, and dispersion in the model numerics. Thus it is understandable that the model data mismatch is greater over the land due to this "representativeness" error (so called because the issue is not that the data are erroneous per se, but rather that they may not represent the observational equivalent of a model grid-box climatology).

With this in mind, we performed some sensitivity analyses into our treatment of the ensembles and the errors, which demonstrate overall that our results appear to be robust to these choices. Focussing first on the issue of model similarity (discussed above in Sect. 2.1) we do find that in terms of correlation and RMS differences, successive generations of models from a single centre, or AO and AOV versions of the same model, are generally more similar to each other than to other models in the sample. Therefore, we first excluded the two PMIP3 models which provide both SAT and SST. This does not make a difference to the level of reliability, a result consistent with the suggestion that CMIP3 and CMIP5 models do not appear to have substantially different behaviour in 
(a) Rank of data in model ensemble.

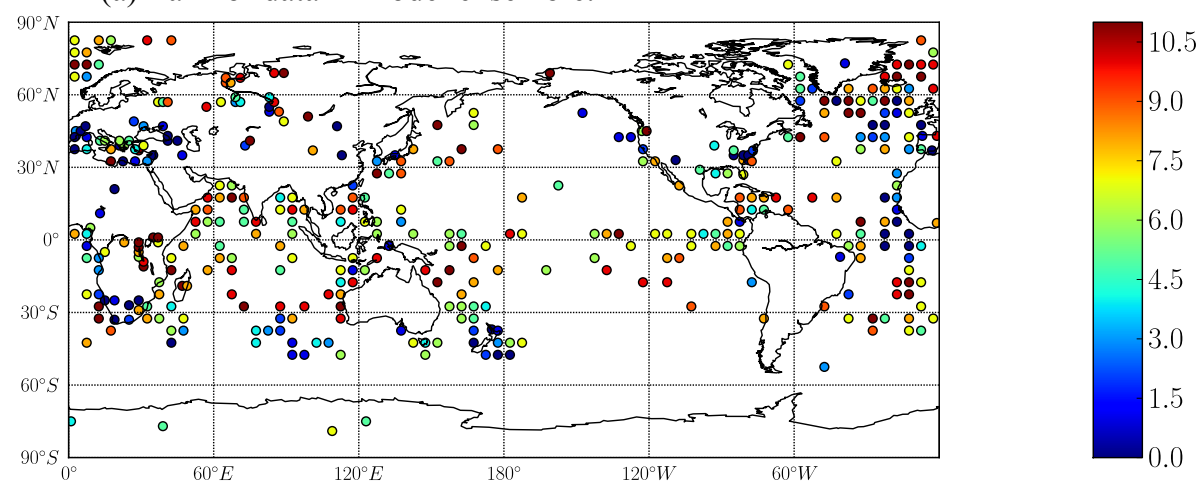

(b) Rank histogram

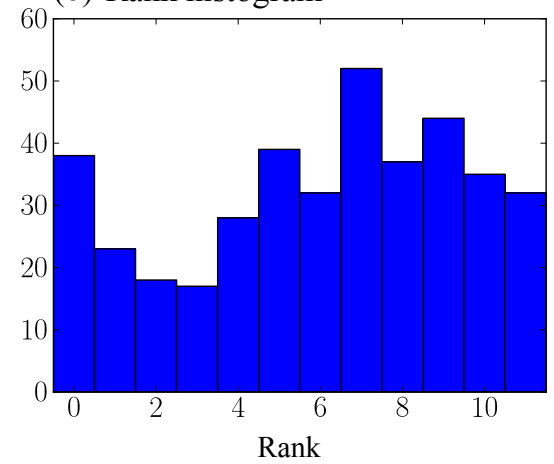

(c) Ensemble mean bias

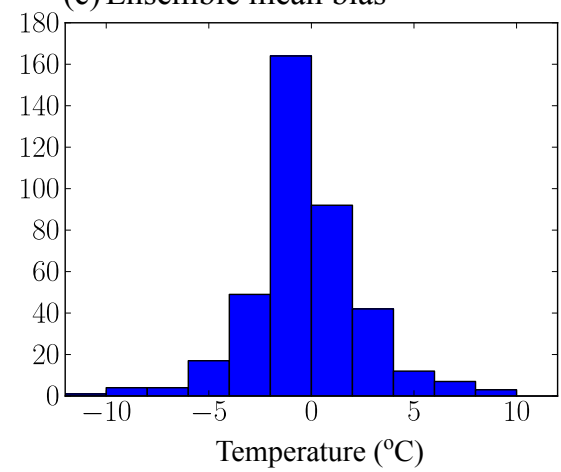

Fig. 3. (a) The rank of the data in the 11 member LGM ensemble. (b) Rank histogram of the ranks in plot (a). (c) The histogram of the difference between the ensemble mean and the data for each data point in plot (a). A low rank indicates that the climate change is greater in the models than the data.

distribution (though with only two PMIP3 models, any difference would be hard to detect in any case). As an alternative to excluding models, we also tested down weighting models which were related in this way, assigning half weight to each model in the pair. In the case of our 11 member ensemble, this means that the PMIP2 and PMIP3 IPSL and ECHAM (MPI) models each had half-weight each as did the PMIP2 HadCM2 AO and AOV models. The results of the rank histogram analysis are not significantly different for those using the whole ensemble equally weighted.

Due to our concerns over the magnitude of the data uncertainties (Annan and Hargreaves, 2013), we also tried an assumption of spatially uniform errors, setting this value to $2{ }^{\circ} \mathrm{C}$ (close to the mean of the individual error estimates). Our results are unchanged by this alteration.

\subsubsection{LGM Skill}

Figure 5 shows the results for the skill calculations for the LGM anomaly. For the PMIP2 and PMIP3 models, we analysed ocean and land both separately and together, and we also calculated the skill for the multi-model mean along with (a) Rank histogram for ocean points
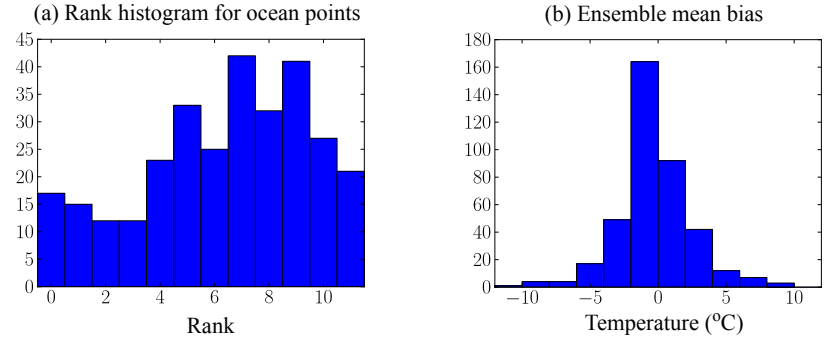

(c) Rank histogram for land points

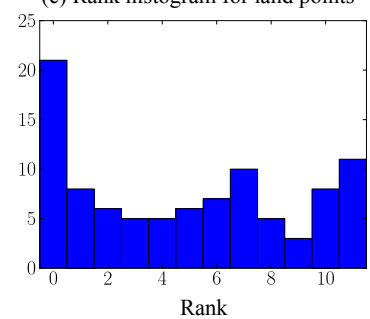

(d) Ensemble mean bias

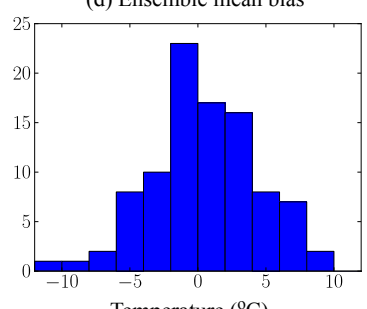

Temperature $\left({ }^{\circ} \mathrm{C}\right)$

Fig. 4. Rank histograms and mean bias histograms for the LGM anomaly, considering the ocean and land separately. 


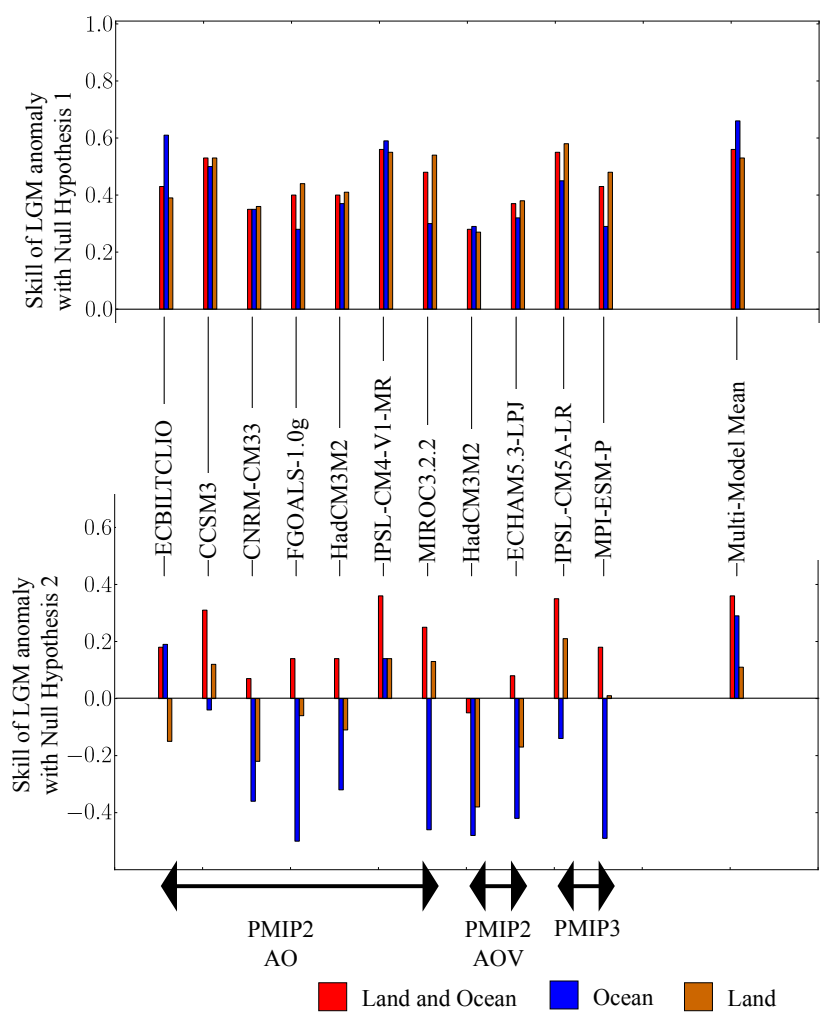

Fig. 5. Skill for the LGM anomaly. The top plot shows the result using the first reference that the LGM anomaly is zero, and the lower plot the results using the second reference that the LGM anomaly is equal to the data mean.

each model individually. For the first reference forecast, of a zero LGM anomaly, there is skill for both the land and the ocean individually, and both combined. As expected (Annan and Hargreaves, 2011) the multi-model mean performs relatively well. Thus we can see that in general the models are producing a cooling that, overall, is of the same scale as the data. As mentioned above, this is, however, a rather limited test. A skill score of 0.5 indicates that the modelled anomalies are typically $50 \%$ greater or smaller than observed.

The second reference forecast is of a uniform field equal to the data mean. This provides a much greater challenge to the models, as they have to not only reproduce a broadscale cooling of the correct magnitude (which the reference forecast already achieves), but must also represent the spatial pattern and magnitude of changes. While on the face of it, this still does not seem like a highly challenging requirement (given the well-known phenomena due to landocean contrast and polar amplification), none of the models have high skill against this reference, and in fact more than half the models have negative skill when assessing the land and ocean separately. Separate assessment of these data sets eliminates the influence of the large land-sea contrast and thereby provides a stiffer challenge. The skill of the multimodel mean is generally greater, especially for the ocean where it outperforms all of the ensemble members, and is also positive for the land. This indicates that the broad scale features which remain after the models are averaged, do bear some relation to the spatial pattern in the data.

The combination of land and ocean together shows much improved skill for all the models compared to the assessments of land and ocean separately. The main reason for this greatly improved performance is that the land-ocean contrast itself is a fairly dominant feature of the climate state which is reasonably well represented in the models, even when they cannot accurately represent the spatial patterns on either land or ocean. This is encouraging, especially in light of recent work (Schmittner et al., 2011) in which an intermediate complexity model appeared to underestimate this land-sea contrast significantly. In the data, the LGM anomaly is larger over the land than the ocean, with the simple averages of the data points (making no attempt to account for spatial distribution) over these regions being $-6.53{ }^{\circ} \mathrm{C}$ and $-1.98^{\circ} \mathrm{C}$, respectively, giving a land-ocean ratio of 3.30 . For the models, the averages over the data points are from $-3.59^{\circ} \mathrm{C}$ to $-9.00{ }^{\circ} \mathrm{C}$ over the land and from $-1.88^{\circ} \mathrm{C}$ to $-2.95^{\circ} \mathrm{C}$ over the ocean, and the land-ocean ratios range from 1.90 to 3.09. So the data ratio is outside the model range, but not necessarily to an alarming degree. It seems plausible that missing forcings (such as dust forcing) may have more effect over land than ocean (Schmittner et al., 2011), which could imply the error is more in boundary conditions rather than models themselves. Overall it must be noted that the levels of skill are not particularly high based on either of the two reference forecasts, suggesting that much of the sub-continental spatial pattern of the change is not being reproduced by the models and that there is plenty of scope for improvement.

\subsubsection{LGM Taylor Diagram}

Conventional statistics are presented in the form of a Taylor diagram in Fig. 6. The modelled LGM anomalies shown in the top plot have correlations with the data which range from around 0.4 to almost 0.7 , with a centred RMS difference which is somewhat lower than the standard deviation of the data itself (which is $3.5^{\circ} \mathrm{C}$ in this case). However, observational errors are quite large, as is indicated by the location of the theoretical "perfect model". The lower plot shows that if instead we had "perfect data" with no observational uncertainty, we could expect the correlations to mostly lie in the range $0.6-0.8$ and the RMS difference from the data would also be substantially smaller.

\subsection{Mid-Holocene analyses - reliability, skill, and Taylor diagrams}

For the MH we analysed eight ensembles. For both the full and conservative ensembles we analysed the temperature anomalies for the annual average, and the hottest and coldest months. For the annual average we made separate analyses 
(a) Conventional Taylor Diagram

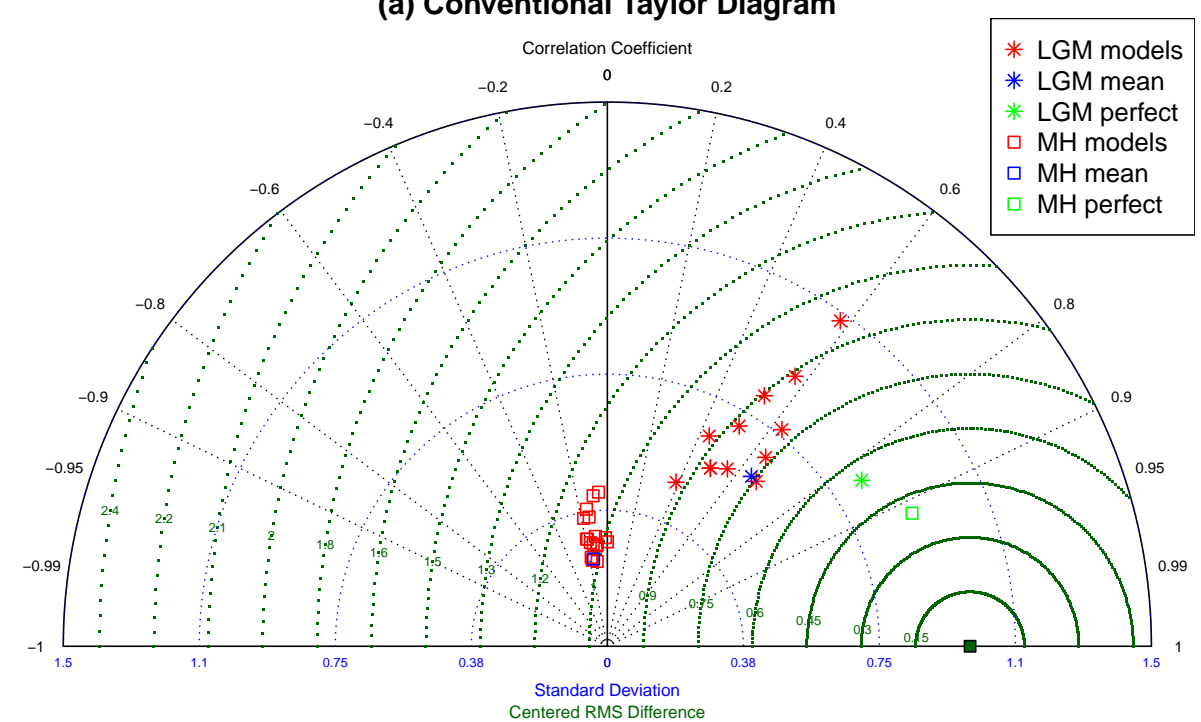

(b) Adjusted Taylor Diagram

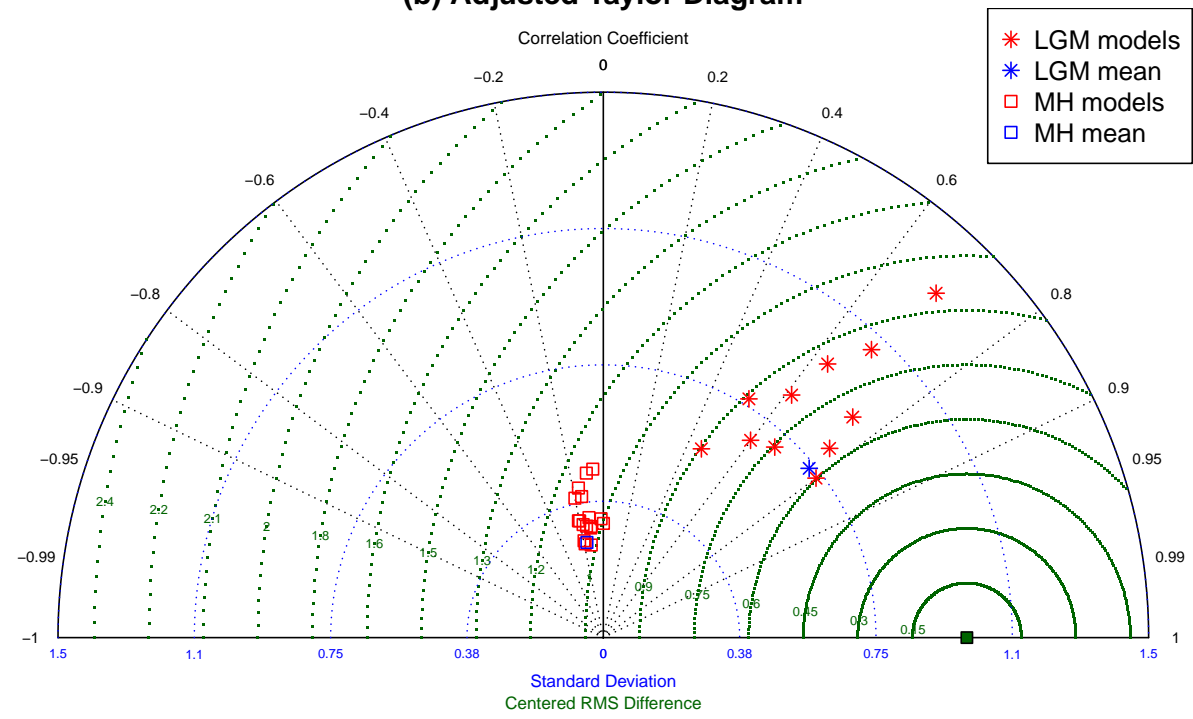

Fig. 6. Taylor diagrams for the LGM mean temperature anomaly and MH hottest month anomaly. Distance from origin indicates standard deviation of field, distance from reference point indicates centred RMS difference between model and data, and pattern correlation is given by the azimuthal coordinate. The left plot shows conventional analysis, with the location of the "perfect model" indicated for comparison. The right plot shows the analysis where model statistics are corrected to account for observational errors. All results are normalised by the standard deviation of the data fields.

with and without including the GHOST ocean data. The results obtained for the $\mathrm{MH}$ are mostly negative for both the reliability and skill analyses. Of all the analyses, only three ensembles are not shown to be unreliable (through significantly non-uniform rank histograms), and even these are visibly tending towards being U-shaped (see Fig. 7). These are the mean temperature anomaly for land and ocean for the conservative ensemble (where we downweight similar models), and the coldest month anomaly for both the full and conservative ensembles. The anomaly for the hottest month is unreliable. This anomaly is also considerably larger in the models than that of the coldest month, and thus although we do not directly test it, we expect that the anomaly in the seasonal cycle is also unlikely to be reliably predicted by the models.

For the skill analyses, the picture is even worse, with most models having negative skill for most target data sets, and no models or model means having skill greater than 0.1 (not shown). There is no indication in the skill results, or in an analysis of the RMS model-data differences, that the AOVGCMs models perform any better than the AOGCMs. This is somewhat disappointing as it is widely thought that vegetation feedbacks had a strong influence on the climate of the MH interval. The best results in these analyses were 

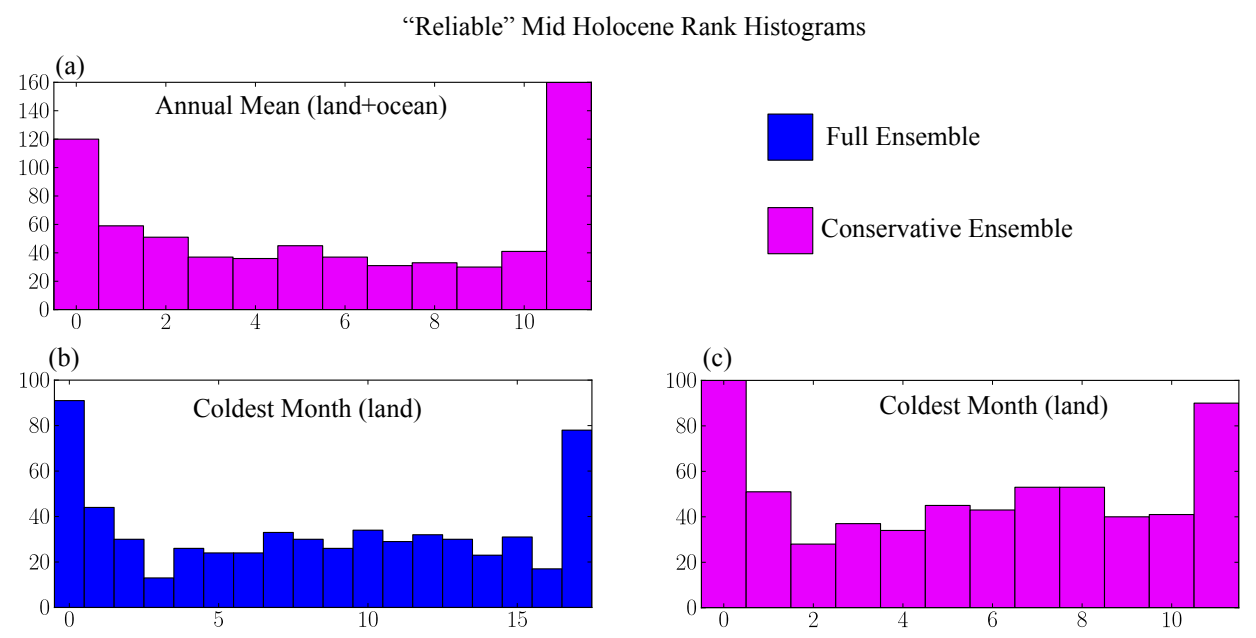

Fig. 7. Rank histograms for the three out of the eight ensembles analysed for the mid-Holocene which passed the statistical test for reliability. They nevertheless show a tendency towards being too narrow.

when we tested ocean (annual mean) data only, and in this case a majority of models exhibited very small positive skill. The Taylor diagram (Fig. 6) also indicates poor results for the $\mathrm{MH}$ hottest month. Correlations are typically negative (albeit small), and the model fields exhibit substantially too small variability. In contrast to the situation for the LGM in Sect. 4.1.3, accounting for observational uncertainty (which is estimated to be relatively small) does not improve these results significantly.

As is the case for the LGM data, some of the observational error estimates appear optimistic, reaching values as low as $0.04{ }^{\circ} \mathrm{C}$. Therefore, we tested the impact of replacing the stated error estimates with a spatially uniform value of $2{ }^{\circ} \mathrm{C}$. However, this barely affects our results.

\section{Discussion}

Our results for the LGM are generally positive, indicating that state of the art climate models are responding to these large changes in boundary conditions in a manner which is not only qualitatively correct, but also quantitatively reasonable. The large-scale cooling simulated by most models has an appropriate magnitude and the pattern shows reasonable agreement with the data, primarily dominated by land-ocean contrast and polar amplification. These results are not surprising, being consistent with (and extending) the analysis of H11, but it may be the first time that this has been shown in a quantitatively detailed manner using comprehensive global data sets. Even for the LGM, however, the sub-continental patterns show weaker agreement with data, as is indicated by the rather poor skill scores relative to the second null hypothesis of a uniform temperature change. On average, the agreement between models and data at the LGM is a little better over the ocean than over land. The high spatial variability in some land data, and limited model resolution, suggests that part of this mismatch may be due to "representativeness" errors.

The MH lacks the strong annual mean forcing that is present at the LGM, and we have seen from the LGM results that the models perform better at the large spatial scales. Thus, we expect the MH interval to provide a greater challenge for the ensemble. The challenge is further increased because, in comparison with the LGM, for the MH we have far more land data points and far fewer ocean points. Thus the "representativeness" error alone could cause the modeldata disagreement to be greater for the MH than LGM. The data are not evenly distributed around the globe at the $\mathrm{MH}$, with high density in Europe and North America, but very poor coverage elsewhere on land and in the oceans.

Consistent with these expectations, we find that the $\mathrm{MH}$ results are substantially poorer than those for the LGM in all respects. Indeed the ensemble is largely unreliable at the MH, with essentially zero (or even negative) skill. Furthermore, it appears possible from examination of the data that there are coherent spatial patterns in reality that are not quantitatively reproduced by the models. To make this point more clearly, we rebinned the data and the multi-model mean for the hottest month into 10 degree boxes. The result is shown in Fig. 8. In Europe and Africa, the anomaly in the observations appears predominantly positive. In North America, the anomalies are smaller and more mixed. The data are, however, generally sparse so it is far from certain whether or not there is a significant spatial pattern in the data. What is clear is that to the extent that there is a pattern in the data across these regions, it is substantially different to that of the multimodel mean response to the forcing. Thus it appears that the model-data mismatch is not just due to sub-grid-scale variability. It should be noted that the models are responding to the applied forcing much in the way that would be expected 

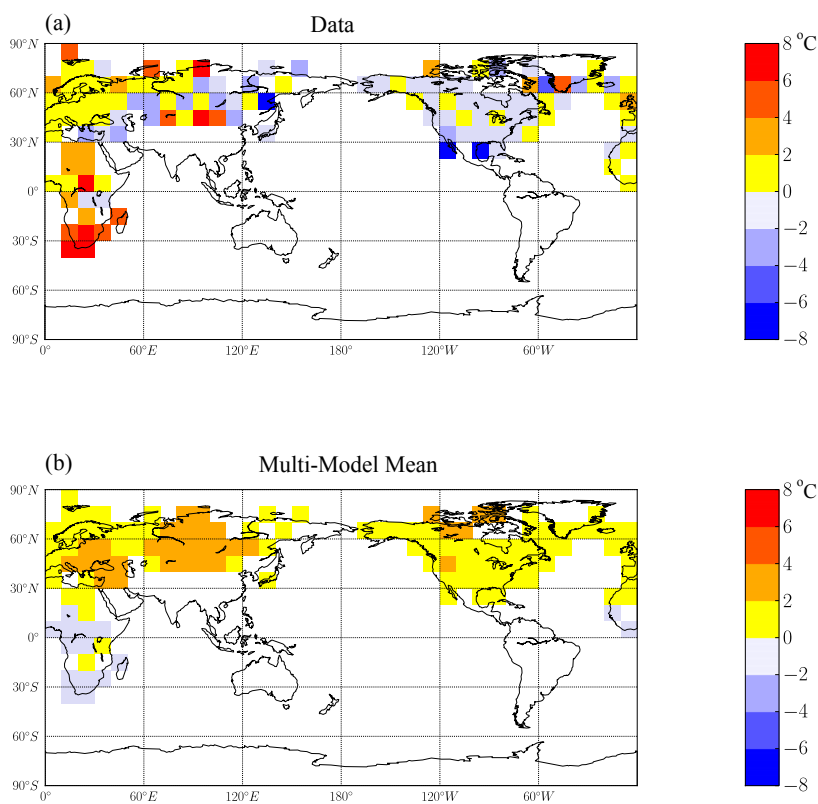

Fig. 8. The hottest month $\mathrm{MH}$ anomaly rebinned onto a 10 degree grid, to more clearly illustrate the model-data mismatch on the subcontinental scale.

from simple physical intuition, with changes in seasonality directly relating to the changes in radiative forcing, and little change in annual mean temperature. Therefore, it seems likely that missing or erroneous feedbacks in the models are contributing to the mismatch. Poor representation of vegetation itself is one possible cause, though it should also be noted that climate models have limited ability to represent fine details of precipitation which, while not directly tested here, will also likely lead to significant errors in vegetation cover. Improved global data coverage should help to clarify the source of model-data mismatch.

While more qualitative approaches have produced some positive results for the MH (Brewer et al., 2007), our direct comparison of gridded data to model output highlights the substantial discrepancies. When considering only ocean data, Schneider et al. (2010) and Lohmann et al. (2012) found positive correlations between model results and proxy data, but also showed their models to greatly underestimate the magnitude of the changes. When we use only ocean data, we find consistent results, with many models showing positive skill for the annual mean temperature change, but only at an extremely low level (typically less than 0.02 in absolute magnitude). The correlations are somewhat larger, averaging 0.3 across the ensemble. Understanding and reducing the discrepancy in magnitude of response between models and data remains a major challenge.

\section{Conclusions}

In this paper we extended our previous analysis of the LGM to include more data, more models, and the MH interval. We also performed the first conventional analysis of predictive skill for paleoclimate GCMs, and present Taylor diagram summaries for both intervals. In these model-data comparison exercises, we have obtained generally positive and encouraging results for the LGM, showing that the models produce generally reasonable and informative predictions of the large-scale response to strong forcing. However, limitations are apparent at finer scales. The model-data mismatch is quite large but it is possible that representativeness error in the data is obscuring the signal, particularly on land.

The MH, with its much smaller net climate forcing, clearly highlights the difficulties of reproducing sub-continental scale patterns of climate change. For this experiment the global climate change signal is very small, and the changes are sub-continental and seasonal in nature, possibly involving significant vegetation feedbacks.

For the point of view of directly using existing models to constrain future climate, the LGM with its large forcing seems the most promising of the two experiments (e.g. Hargreaves et al., 2012). However, climate science is now facing the challenge of predicting future changes on regional scales, which includes the requirement to correctly model vegetation and many other feedbacks. Our results provide some sobering evidence of the limits to the ability of current models to accurately reproduce the local patterns of change that are seen in paleoclimate data. Therefore, unlocking the reasons for the local to regional model-data mismatch for paleoclimates should be a powerful contribution to furthering progress in this area.

Both data and model archives are subject to change. The model output used here were obtained from the model archives in mid-2012. The data were obtained from Andreas Schmittner's website, and directly from Pat Bartlein around the same time. For reasons of reproducibility, we include the derived output from the models, and the data that we used, in the Supplement to this paper.

\section{Supplementary material related to this article is available online at: http://www.clim-past.net/9/811/2013/ cp-9-811-2013-supplement.zip.}

Acknowledgements. We are particularly grateful to Pat Bartlein for his considerable patience while helping us to use the pollen data sets for the LGM and MH, and the GHOST data set for the MH. Two anonymous reviewers also made a large number of helpful suggestions. This work was supported by the S-10-3 project of the MoE, Japan and by the Sousei project of MEXT, Japan. We acknowledge all those involved in producing the PMIP and 
CMIP multi-model ensembles and data syntheses, without which this work would not exist. We acknowledge the World Climate Research Programme's Working Group on Coupled Modelling, which is responsible for CMIP, and we thank the climate modeling groups for producing and making available their model output. For CMIP the US Department of Energy's Program for Climate Model Diagnosis and Intercomparison provides coordinating support and led development of software infrastructure in partnership with the Global Organization for Earth System Science Portals.

Edited by: M. Kageyama

\section{References}

Annan, J. D. and Hargreaves, J. C.: Reliability of the CMIP3 ensemble, Geophys. Res. Lett., 37, L02703, doi:10.1029/2009GL041994, 2010.

Annan, J. D. and Hargreaves, J. C.: Understanding the CMIP3 multimodel ensemble, J. Climate, 24, 4529-4538, 2011.

Annan, J. D. and Hargreaves, J. C.: A new global reconstruction of temperature changes at the Last Glacial Maximum, Clim. Past, 9, 367-376, doi:10.5194/cp-9-367-2013, 2013.

Bartlein, P., Harrison, S., Brewer, S., Connor, S., Davis, B., Gajewski, K., Guiot, J., Harrison-Prentice, T., Henderson, A., Peyron, O., Prentice, I. C., Scholze, M., Seppaä, H., Shuman, B., Sugita, S., Thompson, R. S., Viau, A. E., Williams, J., and Wu, H.: Pollen-based continental climate reconstructions at 6 and $21 \mathrm{ka}$ : a global synthesis, Clim. Dynam., 37, 775-802, 2011.

Braconnot, P., Otto-Bliesner, B., Harrison, S., Joussaume, S., Peterchmitt, J.-Y., Abe-Ouchi, A., Crucifix, M., Driesschaert, E., Fichefet, Th., Hewitt, C. D., Kageyama, M., Kitoh, A., Laîné, A., Loutre, M.-F., Marti, O., Merkel, U., Ramstein, G., Valdes, P., Weber, S. L., Yu, Y., and Zhao, Y.: Results of PMIP2 coupled simulations of the Mid-Holocene and Last Glacial Maximum Part 1: experiments and large-scale features, Clim. Past, 3, 261277, doi:10.5194/cp-3-261-2007, 2007a.

Braconnot, P., Otto-Bliesner, B., Harrison, S., Joussaume, S., Peterchmitt, J.-Y., Abe-Ouchi, A., Crucifix, M., Driesschaert, E., Fichefet, Th., Hewitt, C. D., Kageyama, M., Kitoh, A., Loutre, M.-F., Marti, O., Merkel, U., Ramstein, G., Valdes, P., Weber, L., Yu, Y., and Zhao, Y.: Results of PMIP2 coupled simulations of the Mid-Holocene and Last Glacial Maximum - Part 2: feedbacks with emphasis on the location of the ITCZ and mid- and high latitudes heat budget, Clim. Past, 3, 279-296, doi:10.5194/cp-3-279-2007, 2007b.

Bretherton, C., Widmann, M., Dymnikov, V., Wallace, J., and Bladé, I.: The effective number of spatial degrees of freedom of a timevarying field, J. Climate, 12, 1990-2009, 1999.

Brewer, S., Guiot, J., and Torre, F.: Mid-Holocene climate change in Europe: a data-model comparison, Clim. Past, 3, 499-512, doi:10.5194/cp-3-499-2007, 2007.

Glickman, T.: 2000: Glossary of Meteorology, Am. Meteorol. Soc., 2nd Edn., 2000.
Guiot, J., Boreux, J., Braconnot, P., and Torre, F.: Data-model comparison using fuzzy logic in paleoclimatology, Clim. Dynam., 15, 569-581, 1999.

Hamill, T.: Interpretation of rank histograms for verifying ensemble forecasts, Mon. Weather Rev., 129, 550-560, 2001.

Hargreaves, J. C.: Skill and uncertainty in climate models, Wiley Interdisciplinary Reviews: Climate Change, 1, 556-564, doi:10.1002/wcc.58, 2010.

Hargreaves, J. C., Paul, A., Ohgaito, R., Abe-Ouchi, A., and Annan, J. D.: Are paleoclimate model ensembles consistent with the MARGO data synthesis?, Clim. Past, 7, 917-933, doi:10.5194/cp-7-917-2011, 2011.

Hargreaves, J. C., Annan, J. D., Yoshimori, M., and Abe-Ouchi, A.: Can the Last Glacial Maximum constrain climate sensitivity?, Geophys. Res. Lett., 39, L24702, doi:10.1029/2012GL053872, 2012.

Jolliffe, I. and Primo, C.: Evaluating Rank Histograms Using Decompositions of the Chi-Square Test Statistic, Mon. Weather Rev., 136, 2133-2139, 2008.

Joussaume, S. and Braconnot, P.: Sensitivity of paleoclimate simulation results to season definitions, J. Geophys. Res., 102, 19431956, 1997.

Leduc, G., Schneider, R., Kim, J.-H., and Lohmann, G.: Holocene and Eemian sea surface temperature trends as revealed by alkenone and $\mathrm{Mg} / \mathrm{Ca}$ paleothermometry, Quaternary Sci. Rev., 29, 989-1004, 2010.

Lohmann, G., Pfeiffer, M., Laepple, T., Leduc, G., and Kim, J.H.: A model-data comparison of the Holocene global sea surface temperature evolution, Clim. Past Discuss., 8, 1005-1056, doi:10.5194/cpd-8-1005-2012, 2012.

MARGO Project Members: Constraints on the magnitude and patterns of ocean cooling at the Last Glacial Maximum, Nat. Geosci., 2, 127-132, doi:10.1038/NGEO411, 2009.

Masson, D. and Knutti, R.: Climate model genealogy, Geophys. Res. Lett, 38, L08703, doi:10.1029/2011GL046864, 2011.

Schmittner, A., Urban, N., Shakun, J., Mahowald, N., Clark, P., Bartlein, P., Mix, A., and Rosell-Melé, A.: Climate Sensitivity Estimated from Temperature Reconstructions of the Last Glacial Maximum, Science, 334, 1385-1388, 2011.

Schneider, B., Leduc, G., and Park, W.: Disentangling seasonal signals in Holocene climate trends by satellitemodel-proxy integration, Paleoceanography, 25, PA4217, doi:10.1029/2009PA001893, 2010.

Steig, E.: Mid-Holocene climate change, Science, 286, 1485-1487, 1999.

Taylor, K.: Summarizing multiple aspects of model performance in a single diagram, J. Geophys. Res., 106, 7183-7192, 2001.

Yokohata, T., Annan, J., Collins, M., Jackson, C., Tobis, M., Webb, M. J., and Hargreaves, J. C.: Reliability of multi-model and structurally different single-model ensembles, Clim. Dynam., 39, 599-616, doi:10.1007/s00382-011-1203-1, 2011. 